\title{
Hydromechanics of low-Reynolds-number flow. Part 5. Motion of a slender torus
}

\author{
By ROBERT E. JOHNSON \\ Department of Theoretical and Applied Mechanics, \\ University of Illinois at Urbana-Champaign, Urbana \\ AND THEODORE Y. WU \\ Engineering Science Department, California Institute of Technology, Pasadena
}

(Received 20 February 1979)

In order to elucidate the general Stokes flow characteristics present for slender bodies of finite centre-line curvature the singularity method for Stokes flow has been employed to construct solutions to the flow past a slender torus. The symmetry of the geometry and absence of ends has made a highly accurate analysis possible. The no-slip boundary condition on the body surface is satisfied up to an error term of $O\left(\epsilon^{2} \ln \epsilon\right)$, where $\epsilon$ is the slenderness parameter (ratio of cross-sectional radius to centre-line radius). This degree of accuracy makes it possible to determine the force per unit length experienced by the torus up to a term of $O\left(\epsilon^{2}\right)$. A comparison is made between the force coefficients of the slender torus to those of a straight slender body to illustrate the large differences that may occur as a result of the finite centre-line curvature.

\section{Introduction}

This investigation is concerned with the Stokes flow past bodies possessing finite centre-line curvature. In this paper we consider the flow of a viscous, incompressible, inertialess fluid past a slender torus of circular cross-section, i.e. a torus whose centreline radius $a$ is large compared to its cross-sectional radius $b$. The solution for an arbitrary torus motion will be constructed by applying the method of singularities which was extensively studied for bodies which are not necessarily slender by Chwang $\& W u(1974,1975)$. By distributing appropriate stokeslets, doublets, rotlets, sources, stresslets, and quadrupoles on the body centre-line the no-slip boundary condition on the torus surface is satisfied analy tically in closed form neglecting terms of $O\left(\epsilon^{2} \ln \epsilon\right)$, where $\epsilon=b / a$.

Previous solution methods for slender bodies with curved centre-lines (Cox 1970; Keller \& Rubinow 1976) have employed the method of matched asymptotic expansions with the inner expansion being the flow past a straight circular cylinder. Here no such approximation is made and the effect of local centre-line curvature is retained. Furthermore, because of the analytic complexity in the previous methods, it is only practical to obtain results for the force on curved centre-line bodies whose error is $O(1 / \ln \epsilon)^{3}$. In the present study considerable improvement has been made by obtaining the force with an error of $O\left(\epsilon^{2}\right)$. This result has essentially summed the infinite series involving powers of $1 / \ln \epsilon$. 
The principal results found in this paper have been briefly summarized by Wu (1976, 1977) for application in biomechanics. It is also worthwhile to note that the solution for the torus has served as a useful guide in constructing a solution, possessing the same accuracy, for slender bodies of arbitrary centre-line configuration (Johnson 1977). That problem will appear shortly in a separate paper.

\section{Motion of a slender torus}

For the motion of a body with surface $S_{b}$ in an incompressible, inertialess fluid the velocity $u$ and pressure $p$ in the fluid satisfy the Stokes equations

$$
\nabla p=\mu \nabla^{2} \mathbf{u}, \quad \nabla \cdot \mathbf{u}=0
$$

along with the no-slip boundary conditions

$$
\begin{aligned}
& \mathbf{u}=\mathbf{U} \quad \text { for } \mathbf{x} \text { on } S_{b}, \\
& \mathbf{u} \rightarrow 0 \text { as } \mathbf{x} \rightarrow \infty,
\end{aligned}
$$

$\mu$ being the fluid viscosity, $\mathbf{U}$ the velocity of the body surface and $\mathbf{x}$ the position vector in a three-dimensional Euclidean space.

The linearity of the Stokes equations allow us to decompose the arbitrary motion of a torus into five fundamental problems: a translation along and a translation perpendicular to the generating or longitudinal axis, a rotation about an axis perpendicular to the longitudinal axis and intersecting the torus centre-line, a rotation about the longitudinal axis, and a torus expanding radially. If we take the longitudinal axis to be coincident with the $z$ axis of a Cartesian co-ordinate system (figure 1 ) the general torus motion described above may be written as

$$
\mathbf{U}=U_{z} \mathbf{e}_{z}+U_{x} \mathbf{e}_{x}+U_{y} \mathbf{e}_{y}+\boldsymbol{\omega} \times\left(a \mathbf{e}_{r}+b \mathbf{e}_{r_{1}}\right)+U_{r} \mathbf{e}_{r},
$$

where $\omega$ is the angular velocity. In terms of the cylindrical co-ordinate $(r, \theta, z)$ the corresponding velocity components $(u, v, w)$ are given by

$$
\begin{gathered}
u=U_{y} \sin \theta+U_{x} \cos \theta+U_{r}+\epsilon \sin \psi\left(a \omega_{y} \cos \theta-a \omega_{x} \sin \theta\right), \\
v=U_{y} \cos \theta-U_{x} \sin \theta+a \omega_{z}(1+\epsilon \cos \psi)-\epsilon \sin \psi\left(a \omega_{y} \sin \theta+a \omega_{x} \cos \theta\right), \\
w=U_{z}+\left(a \omega_{x} \sin \theta-a \omega_{y} \cos \theta\right)(1+\epsilon \cos \psi) .
\end{gathered}
$$

Guided by the results found for slender bodies with straight centre-lines we represent the flow field, as a first approximation, by a distribution of stokeslets and doublets along the body centre-line $r=a$. The velocity at $\theta=\theta^{\prime}$ is therefore given by

$$
\mathbf{u}=\int_{\theta^{\prime}}^{\theta^{\prime}+2 \pi}\left[\mathbf{U}_{\mathrm{s}}(\mathbf{R} ; \boldsymbol{\alpha})+\mathbf{U}_{\mathrm{d}}(\mathbf{R} ; \boldsymbol{\beta})\right] a d \theta
$$

where the stokeslet and doublet velocity fields are given respectively by

$$
\begin{aligned}
\mathrm{U}_{\mathrm{s}} & =\frac{\alpha}{R}+\frac{(\alpha \cdot \mathbf{R}) \mathbf{R},}{R^{3}}, \\
\mathrm{U}_{\mathrm{d}} & =\frac{\beta}{R^{3}}-\frac{3(\beta \cdot \mathbf{R}) \mathbf{R}}{R^{5}},
\end{aligned}
$$




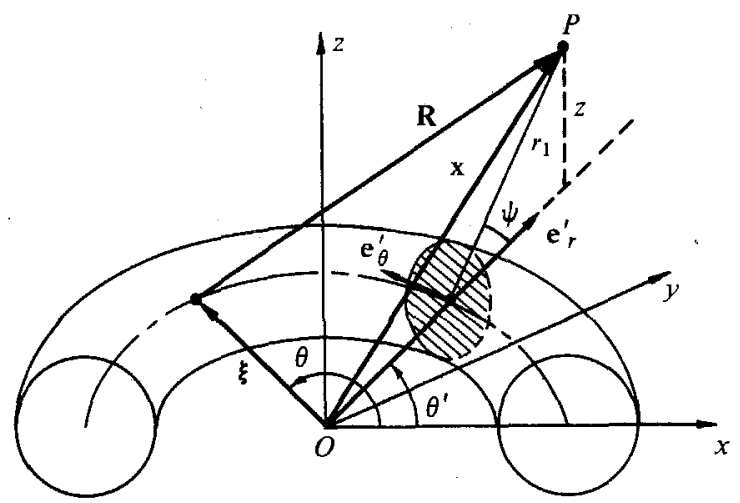

(a)

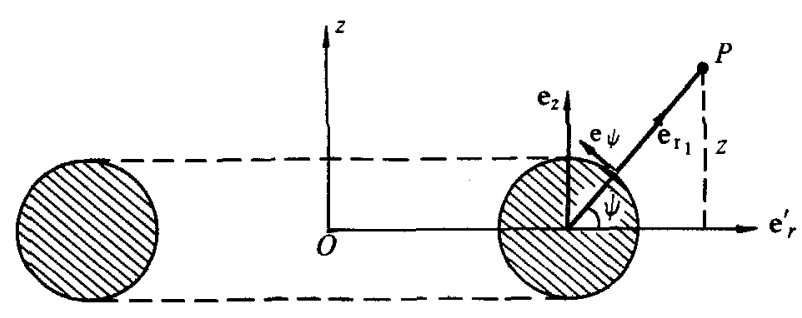

(b)

FigURE 1. (a) Torus and co-ordinates. (b) Sectional view of torus at $\theta^{\prime}=$ constant.

and noting figure 1,

$$
\begin{aligned}
& \mathbf{R}=\mathbf{x}-\xi=\left(r \cos \theta^{\prime}-a \cos \theta\right) \mathbf{e}_{x}+\left(r \sin \theta^{\prime}-a \sin \theta\right) \mathbf{e}_{y}+z \mathbf{e}_{z}, \\
& R=|\mathbf{R}|=\left(r^{2}+a^{2}+z^{2}-2 a r \cos \phi\right)^{\frac{1}{2}}, \\
& \phi=\theta-\theta^{\prime} .
\end{aligned}
$$

In terms of the unit vectors $\mathbf{e}_{r}^{\prime}, \mathbf{e}_{\theta}^{\prime}, \mathbf{e}_{z}$ at $\theta=\theta^{\prime}$ (see figure 1) we write

$$
\mathbf{R}=(r-a \cos \phi) \mathbf{e}_{\tau}^{\prime}-a \sin \phi \mathbf{e}_{\theta}^{\prime}+z \mathbf{e}_{z} .
$$

Furthermore, by introducing the curvilinear orthogonal co-ordinate system $\left(r_{1}, \theta, \psi r\right)$ defined by

we have

$$
\begin{aligned}
& x=r \cos \theta=\left(a+r_{1} \cos \psi\right) \cos \theta \\
& y=r \sin \theta=\left(a+r_{1} \cos \psi\right) \sin \theta \\
& z=r_{1} \sin \psi
\end{aligned}
$$

$$
R=\left[2 a^{2}\left(1+\frac{r_{1}}{a} \cos \psi\right)(1-\cos \phi)+r_{1}^{2}\right]^{\frac{1}{2}} \text {. }
$$

From the flow and body symmetry and the fact that the stokeslet strength $\alpha$ is proportional to the force per unit length experienced by the body (i.e. $f=-8 \pi \mu \alpha$ ) 
we assume $\alpha$ priori that $\alpha$ is proportional to the body centre-line velocity. Thus noting (1), (2) and (3) we take the components of the stokeslet strength in the cylindrical co-ordinate system to be

$$
\left.\begin{array}{l}
\alpha_{r}=K_{r}^{(1)} \sin \theta+K_{r}^{(2)} \cos \theta+K_{r}^{(3)}, \\
\alpha_{\theta}=K_{\theta}^{(1)} \cos \theta-K_{\theta}^{(2)} \sin \theta+K_{\theta}^{(3)}, \\
\alpha_{z}=K_{z}^{(1)} \sin \theta-K_{z}^{(2)} \cos \theta+K_{z}^{(3)},
\end{array}\right\}
$$

where the $K$ 's are constants to be determined from the boundary conditions. Furthermore, the results available for straight slender bodies suggest that for each component of the stokeslet oriented normal to the body centre-line there corresponds a doublet also proportional to the centre-line velocity. Therefore we assume that the doublet strength $\beta$ is given by

$$
\left.\begin{array}{l}
\beta_{r}=B_{r}^{(1)} \sin \theta+B_{r}^{(2)} \cos \theta+B_{r}^{(3)} \\
\beta_{\theta}=0, \\
\beta_{z}=B_{z}^{(1)} \sin \theta-B_{z}^{(2)} \cos \theta+B_{z}^{(3)} .
\end{array}\right\}
$$

The above assumptions on the functional form of the singularity distributions will be verified upon satisfying the boundary conditions.

In order to satisfy the no-slip boundary condition on the torus surface $\left(r_{1}=b\right)$ the integrals in (4) must be evaluated, and therefore a general discussion of the methods available is presented. Firstly, for the special case of a torus, where it is possible to presuppose the functional form of the singularity distributions, the integrals in (4) may be expressed in terms of complete elliptic integrals, as was shown by Wu \& Yates (1976) for the analogous potential flow problem. The well-known asymptotic expansions for the elliptic integrals can then be used to evaluate the velocity on the torus surface, $r_{1}=b$. An alternative method which can be applied to general centre-line configurations where the functional forms of the singularity distributions are not known a priori is presented below. Although both methods were used in calculating the results presented here, we will restrict our attention to the latter for two reasons: (1) it can be used for arbitrary body shapes to be discussed in a future paper; (2) a similar elliptic integral method is available elsewhere (Wu \& Yates 1976).

For the torus problem it is sufficient to consider the integral

$$
I=\int_{\theta^{\prime}}^{\theta^{\prime}+2 \pi} \frac{f(\theta)}{R} d \theta
$$

where $R$ is given in (8) and $f(\theta)$ is continuously differentiable for as many times as necessary. Similar integrals arising for the torus problems and those to be found when considering the general slender body case can be evaluated by following the same basic procedure as that to be developed here. The objective is to expand the integrand in equation (11) in a neighbourhood of the body surface, i.e. $r_{1} \ll a$, with the resulting expansion being easily integrated termwise.

The integral above can be written as

$$
I=(a r)^{-\frac{1}{2}} \int_{0}^{\pi} \frac{f\left(\theta^{\prime}+\phi\right)+f\left(\theta^{\prime}-\phi\right)}{\left[2(1-\cos \phi)+r_{1}^{2} / a r\right]^{\frac{1}{2}}} d \phi,
$$


where $r=a+r_{1} \cos \psi$. In the neighbourhood of the torus surface, $r_{1} / a=O(\epsilon)$ and $r_{1}^{2} / a r=O\left(\epsilon^{2}\right)$. Consequently, if $f(\theta)$ is a sufficiently smooth function of $O(1)$, we clearly see that the integrand is of order $\epsilon^{-1}$ at $\phi=0$ and decreases monotonically and rather rapidly to $O(1)$ when $\phi$ moves away from $\phi=0$. We note that the two terms in the square root, namely $1-\cos \phi$ and $r_{1}^{2} / a r$, become of the same order when $\phi$ is of $O(\varepsilon)$. This suggests that we construct an inner expansion of the integrand near $\phi=0$ and an outer expansion for $\phi=O(1)$. For this purpose we introduce the inner or stretched variable

$$
\sigma=\phi / \epsilon
$$

for expansion of the integrand in the 'inner region' near $\phi=0$. We can then construct a uniformly valid or composite expansion from the inner expansion about $\phi=0$ and the outer expansion for $\phi=O(1)$, using the standard methods of perturbation theory (Kaplun 1967; Van Dyke 1975; Cole 1968).

For convenience we define

$$
h\left(\phi, \theta^{\prime} ; \epsilon\right)=\frac{f\left(\theta^{\prime}+\phi\right)+f\left(\theta^{\prime}-\phi\right)}{\left[2(1-\cos \phi)+\epsilon^{2} \rho^{2}\right]^{\frac{1}{2}}},
$$

where $\epsilon^{2} \rho^{2}=r_{1}^{2} / a r$. The inner expansion of $h$ to $n$ terms is then given for $\epsilon \rightarrow 0$, holding $\sigma$ fixed, by

$$
E_{n}^{(\mathrm{i})} h=\lim _{\substack{\epsilon \rightarrow 0 \\ \sigma \text { fixed }}} h\left(\epsilon \sigma, \theta^{\prime} ; \epsilon\right)=\sum_{j=0}^{n-1} \mu_{j}(\epsilon) h_{j}\left(\sigma, \theta^{\prime}\right),
$$

where the coefficients $\mu_{j}(\epsilon)$ form an asymptotic sequence of functions such that $\mu_{n+1}(\epsilon)=o\left(\mu_{n}(\epsilon)\right)$ as $\epsilon \rightarrow 0$. For $\epsilon \rightarrow 0$, with $\phi$ held fixed, we express the outer expansion of $h$ up to $n$ terms as

$$
E_{n}^{(\mathrm{o})} h=\lim _{\substack{\epsilon \rightarrow 0 \\ \phi \text { fixed }}} h\left(\phi ; \theta^{\prime}, \epsilon\right)=\sum_{j=0}^{n-1} \nu_{j}(\epsilon) g_{j}\left(\phi ; \theta^{\prime}\right),
$$

where $\nu_{j}(\epsilon)$ form another asymptotic sequence of functions, namely $\nu_{j+1}(\epsilon)=o\left(\nu_{j}(\epsilon)\right)$ as $\epsilon \rightarrow 0$. In our case we will in fact find $\mu_{j}(\epsilon)=\epsilon^{2 j-1}, v_{j}(\epsilon)=\epsilon^{2 j}$, by virtue of $h$ being even in $\phi$. The uniformly valid expansion is then formed in the usual manner and is given by

$$
E_{n}^{(\mathrm{c})} h=E_{n}^{(\mathrm{i})} h+E_{n}^{(\mathrm{o})} h-E_{n}^{(\mathrm{i})} E_{n}^{(\mathrm{o})} h .
$$

The last term represents an inner expansion and outer expansion operated on $h$ in succession, regardless of order, and is often referred to as the common-part expansion. This uniformly valid expansion as the name implies, holds uniformly for $0 \leqslant \phi \leqslant \pi$, with error of order $\epsilon^{2 n-1}$. Substituting (15) into (12) then allows termwise integration, resulting in an asymptotic expansion for $I$ as $\epsilon \rightarrow 0$. We note that the integration will modify the error of the final result.

The algebra involved in constructing expansion (15) is quite complex but can be reduced for the case of a torus, where the body shape has no ends, by following a somewhat simplified procedure. We write the integral $I$ as follows:

$$
I=(a r)^{-\frac{1}{2}}\left\{\int_{0}^{\gamma} E_{n}^{(\mathrm{i})} h d \phi+\int_{\gamma}^{\pi} E_{n}^{(\mathrm{o})} h d \phi+O\left(\epsilon^{2 n-1}\right)\right\}
$$

with $\gamma$ chosen to be $\epsilon \ll \gamma \ll \pi$. At the level of approximation of interest in the present study, i.e. retaining terms through $O(\epsilon)$ in the integrand, a useful choice is to restrict 
$\gamma$ to $O(1)>O\left(\gamma^{2}\right)>O(\epsilon)$. The above integration procedure is justified by considering the uniformly valid expansion of $h$. We regroup the terms in (15) as follows .

where

$$
\begin{aligned}
E_{n}^{(\mathrm{c})} h & =E_{n}^{(\mathrm{i})} h+R_{n}^{(\mathrm{i})} h, \quad 0 \leqslant \phi \leqslant \gamma, \\
& =E_{n}^{(\mathrm{o})} h+R_{n}^{(\mathrm{o})} h, \quad \gamma \leqslant \phi \leqslant \pi,
\end{aligned}
$$

$$
\begin{aligned}
& R_{n}^{(\mathrm{i})} h=E_{n}^{(\mathrm{o})} h-E_{n}^{(\mathrm{i})} E_{n}^{(\mathrm{o})} h \\
& R_{n}^{(\mathrm{o})} h=E_{n}^{(\mathrm{i})} h-E_{n}^{(\mathrm{o})} E_{n}^{(\mathrm{i})} h
\end{aligned}
$$

We note that both 'residual expansions' $R_{n}^{(\mathrm{i})} h$ and $R_{n}^{(\mathrm{o})} h$ will be of higher order to those terms retained, i.e. $O\left(\epsilon^{2 n-1}\right)$, in their respective regions of $\phi$. It therefore follows that

$$
\begin{aligned}
\int_{0}^{\pi} h d \phi & =\int_{0}^{\pi} E_{n}^{(\mathrm{c})} h d \phi+\int_{0}^{\pi}\left(h-E_{n}^{(\mathrm{c})} h\right) d \phi \\
& =\int_{0}^{\gamma}\left(E_{n}^{(\mathrm{i})} h+R_{n}^{(\mathrm{i})} h\right) d \phi+\int_{\gamma}^{\pi}\left(E_{n}^{(\mathrm{o})} h+R_{n}^{(\mathrm{o})} h\right) d \phi+O\left(\epsilon^{2 n-1}\right) \\
& =\int_{0}^{\gamma} E_{n}^{(\mathrm{i})} h d \phi+\int_{\gamma}^{\pi} E_{n}^{(\mathrm{o})} h d \phi+O\left(\epsilon^{2 n-1}\right) .
\end{aligned}
$$

The resulting expansion of $I$ will not involve $\gamma$ since after integration those terms will cancel. For the general slender body case such a reduction of algebra is not possible and the uniformly valid expansion must be computed in order to construct a solution valid everywhere, including the body ends. We also note that the composite expansion method enables one to distinguish between the short- and long-range contributions to the velocity field, whereas the elliptic integral technique does not readily offer such physical information about the solution.

Returning now to the motion of a torus we separate equation (4) as just discussed:

$$
\mathbf{u}=\int_{\theta^{\prime}+\gamma}^{\theta^{\prime}+2 \pi-\gamma}\left(\mathbf{U}_{\mathrm{s}}+\mathbf{U}_{\mathrm{d}}\right) a d \theta+\int_{\theta^{\prime}-\gamma}^{\theta^{\prime}+\gamma}\left(\mathbf{U}_{\mathrm{s}}+\mathbf{U}_{\mathrm{d}}\right) a d \theta
$$

With respect to the region of integration in the above two integrals we will refer to them as follows: the first will be called the far-field singularity distribution and the second the near-field singularity distribution. The singularity strengths in (16) are determined by applying the no-slip boundary conditions (1), (2), (3) at $\theta=\theta^{\prime}$.

For use in constructing the appropriate expansions to the integrands in (16) we have the inner and outer expansions of $R$ and $\mathbf{R}$ valid in the vicinity of the body surface given by

$$
\begin{aligned}
& R=\sqrt{2} a(1+\epsilon \eta \cos \psi)^{\frac{1}{2}}(1-\cos \phi)^{\frac{1}{2}}\left\{1+O\left(\frac{\epsilon^{2} \eta^{2}}{1-\cos \phi}\right)\right\}, \\
& \mathbf{R}=a\left\{(1-\cos \phi+\epsilon \eta \cos \psi) \mathbf{e}_{r}^{\prime}-\sin \phi \mathbf{e}_{\theta}^{\prime}+\epsilon \eta \sin \psi \mathbf{e}_{z}\right\},
\end{aligned}
$$

for $\gamma<\phi<2 \pi-\gamma$ and

$$
\begin{gathered}
R=a \Delta\left\{1-\frac{\phi^{4}}{24 \Delta^{2}}+O\left(\frac{\epsilon \phi^{4}}{\Delta^{2}}, \frac{\phi^{6}}{\Delta^{2}}\right)\right\}, \\
\mathbf{R}=a\left\{\left(\epsilon \eta \cos \psi+\frac{1}{2} \phi^{2}+O\left(\phi^{4}\right)\right) \mathbf{e}_{r}^{\prime}-\left(\phi-\frac{1}{6} \phi^{3}+O\left(\phi^{5}\right)\right) \mathbf{e}_{\theta}^{\prime}+\epsilon \eta \sin \psi \mathbf{e}_{z}\right\}
\end{gathered}
$$


for $-\gamma<\phi<\gamma$, where $\phi=\theta-\theta^{\prime}, \eta=r_{1} / b$ and $\Delta^{2}=(1+\epsilon \eta \cos \psi) \phi^{2}+(\epsilon \eta)^{2}$. Similarly the inner expansion of the singularity strengths is found by expanding (9) and (10) about the point $\phi=0$, while the outer expansions are given directly by (9) and (10).

Examination of the far-field singularity distribution (i.e. the outer expansion) shows that the contribution to the velocity field near the torus surface at $\theta=\theta^{\prime}$ due to the doublet distribution will be of order $\beta / a^{2}$. Anticipating that the doublet strength to leading order will be the same as for a straight slender body (as will be verified later), namely $\beta$ will be proportional to $\alpha b^{2}$, we conclude that the doublets far-field contribution to the velocity will be of $O\left(\alpha \epsilon^{2}\right)$. For this reason the far-field doublet distribution may be neglected at the present level of approximation. Consequently equation (16) becomes

$$
\mathbf{u}=\int_{\gamma}^{2 \pi-\gamma} \mathbf{U}_{\mathrm{s}}^{(0)} a d \phi+\int_{-\gamma}^{\gamma}\left(\mathbf{U}_{\mathrm{s}}+\mathbf{U}_{\mathrm{d}}\right)^{(\mathrm{i})} a d \phi+O\left(\boldsymbol{\alpha} \epsilon^{2}\right)
$$

where the (i) and (o) refer to the inner and outer expansions. For convenience we now examine each fundamental mode of torus motion separately.

\section{Broadwise translation}

For translation along the longitudinal axis, here referred to as broadwise translation, we have in (1), (2) and (3) $U_{r}=U_{x}=U_{y}=\omega=0$ and $U_{z} \neq 0$. Consequently the only non-zero terms needed in the stokeslet and doublet strengths [equations (9) and (10)] are $K_{z}^{(3)}$ and $B_{z}^{(3)}$. The inner and outer expansions of the integrands are easily computed using (5), (6), (17), (18), (19) and (20), which after integration gives the velocity field on the torus surface $r_{1}=b$ neglecting terms of $O\left(\epsilon^{2}\right)$ (for the sake of brevity the algebraic details are omitted and the reader is referred to Johnson 1977),

$$
\left.\begin{array}{rl}
u= & 2 \cos \psi \sin \psi G_{z}-\epsilon \sin \psi\left[K_{z}(1-L)+\left(B_{z} / b^{2}\right)+\cos ^{2} \psi G_{z}\right], \\
v= & 0, \\
w= & 2 K_{z} L+\left(2 B_{z} / b^{2}\right)+2 \sin ^{2} \psi G_{z}+\epsilon \cos \psi\left[K_{z}(1-L)\right. \\
\quad & \left.\quad\left(B_{z} / b^{2}\right)-\sin ^{2} \psi G_{z}\right],
\end{array}\right\}
$$

where $L=\ln (8 / \epsilon), G_{l}=K_{l}-2 B_{l} / b^{2}(l=r, \theta, z)$ and we have omitted the superscript on $K_{z}$ and $B_{z}$. The no-slip boundary condition on the torus surface $r_{1}=b$, i.e. $u=v=0$ and $w=U_{z}$, can be satisfied to leading order, neglecting terms of $O(\alpha \in \ln \epsilon)$, by taking

$$
B_{z}=\frac{1}{2} K_{z} b^{2}, \quad K_{z}=U_{z} / 2\left[\ln (8 / \epsilon)+\frac{1}{2}\right] .
$$

As previously anticipated the doublet strength is proportional to $b^{2} K_{z}$.

With the stokeslet and doublet strength given by equations (23) the remaining higherorder terms in the velocity field (22) which do not satisfy the no-slip condition may be written using the $\left(r_{1}, \theta, \psi\right)$ co-ordinate system as

$$
\mathbf{u}=-K_{z} \epsilon\left\{(L-1) \mathbf{e}_{\psi}+\frac{1}{2}\left(\sin 2 \psi \mathbf{r}_{r_{1}}+\cos 2 \psi \mathbf{e}_{\psi}\right)\right\} .
$$

Further satisfaction of the boundary condition will clearly require that other fundamental singularities be incorporated into our solution. Noting that the first term in equation (24) is a 'rotational-like' flow in the cross-sectional plane about the torus surface suggests that the addition of a rotlet oriented along the torus centre-line will cancel this term and satisfy the boundary condition through terms of $O(\epsilon \ln \epsilon)$. The 
remaining terms in (24) describe 'extensional-like' flow near the body surface in the cross-section plane. Motivated by the exact solution for a circular cylinder in an extensional flow (Chwang \& Wu 1975) we introduce a stresslet-quadrupole pair into our solution. Both terms in (24) are a manifestation of the local centre-line curvature since they tend to increase the velocity in the $z$ direction on the inside surface of the torus $\left(\frac{1}{2} \pi<\psi<\frac{3}{2} \pi\right)$ and decrease it on the outside surface $\left(-\frac{1}{2} \pi<\psi<\frac{1}{2} \pi\right)$, where the distance from singularity distribution to torus surface is greater.

The velocity field produced by the additional singularity distributions required is

$$
\mathbf{u}=\int_{0}^{2 \pi}\left\{A \mathbf{U}_{\mathbf{r}}\left(\mathbf{R} ; \mathbf{e}_{\theta}\right)+B \mathbf{U}_{\mathrm{ss}}\left(\mathbf{R} ; \mathbf{e}_{r}, \mathbf{e}_{z}\right)+C \mathbf{U}_{\mathrm{q}}\left(\mathbf{R} ; \mathbf{e}_{r}, \mathbf{e}_{z}\right)\right\} a d \phi
$$

where $A, B$ and $C$ are constants and the general form of the rotlet, stresslet and quadrupole velocity fields are respectively given by

$$
\left.\begin{array}{c}
\mathbf{U}_{\mathrm{r}}(\mathbf{R} ; \boldsymbol{\delta})=\frac{\delta \times \mathbf{R}}{R^{3}}, \\
\mathbf{U}_{\mathrm{ss}}(\mathbf{R} ; \mathbf{a}, \mathbf{b})=\left\{-\frac{\mathbf{a} \cdot \mathbf{b}}{R^{3}}+\frac{3(\mathbf{a} \cdot \mathbf{R})(\mathbf{b} \cdot \mathbf{R})}{R^{5}}\right\} \mathbf{R}, \\
\mathbf{U}_{\mathbf{q}}(\mathbf{R} ; \mathbf{a}, \mathbf{b})=-\nabla \Phi ; \quad \Phi=(\mathbf{b} \cdot \nabla)(\mathbf{a} \cdot \nabla)(1 / R) .
\end{array}\right\}
$$

Constructing the inner and outer expansions to the integrand in (25) and integrating as discussed gives for $r_{1}=b$,

$$
\left.\begin{array}{l}
u=\frac{2 \sin \psi}{b}\left[A+B+\frac{2 C}{b^{2}}+\left(B+\frac{4 C}{b^{2}}\right) \cos 2 \psi\right] \\
v=0 \\
w=\frac{2 \cos \psi}{b}\left[-A+B+\frac{2 C}{b^{2}}-\left(B+\frac{4 C}{b^{2}}\right) \cos 2 \psi\right]
\end{array}\right\}
$$

where terms of $O\left(A \epsilon \ln \epsilon, B \epsilon, C \epsilon / b^{2}\right)$ have been neglected within the brackets. We note that in determining (26) only the near-field distributions of these higher-order singularities contribute to the velocity field at this level of approximation and as will always be the case here only the stokeslet, with its long-range effect, has a far-field term which must be retained.

Equating (26) to the remaining higher-order terms in (22), or equivalently (24), gives

$$
A=\frac{1}{2} \epsilon[1-\ln (8 / \epsilon)] b K_{z}, \quad B=\frac{1}{2} \epsilon b K_{z}, \quad C=-\frac{1}{4} b^{2} B=-\frac{1}{8} \epsilon b^{3} K_{z} .
$$

From the above we see that the neglected terms in (26) are of higher order than those retained and we have satisfied the no-slip condition neglecting terms of $O\left(\epsilon^{2} \ln \epsilon\right)$. Furthermore, the force per unit length experienced by the torus is given by

$$
f_{y}=-8 \pi \mu K_{z}=\frac{-4 \pi \mu U_{z}}{\ln (8 / \epsilon)+\frac{1}{2}}+O\left(\epsilon^{2}\right) .
$$

In addition, a torque per unit length about the torus centre-line, $M_{\theta}$, results from the local centre-line curvature effect and is given by

$$
M_{\theta}=2 \pi \mu b \epsilon[2 \ln (8 / \epsilon)-1] K_{z} .
$$


The details of this rather lengthy calculation, which involves integrating the shear stress on the body surface, is given by Johnson (1977).

\section{Translation perpendicular to the longitudinal axis}

For this case it is sufficient to consider only $U_{y}$ non-zero in (1), (2) and (3) (the results being equally applicable for $U_{x} \neq 0$ ). The stokeslet and doublet strengths are therefore given by (9) and (10):

$$
\begin{aligned}
& \alpha_{r}=K_{r} \sin \theta, \quad \alpha_{\theta}=K_{\theta} \cos \theta, \quad \alpha_{z}=0, \\
& \beta_{r}=B_{r} \sin \theta, \quad \beta_{\theta}=0, \quad \beta_{z}=0 .
\end{aligned}
$$

Expanding the integrands in (21) and integrating leads to the following boundary conditions, neglecting terms of $O\left(\epsilon^{2}\right)$, at $\theta=\theta^{\prime}$,

$$
\left.\begin{array}{rl}
U_{v} \sin \theta^{\prime}= & \sin \theta^{\prime}\left\{2 K_{r}(L-1)+4 K_{\theta}+2 B_{r} / b^{2}+2 \cos ^{2} \psi G_{r}\right. \\
\left.+2 \cos \psi \epsilon\left[L\left(K_{\theta}-\frac{1}{2} K_{r}\right)+K_{r}-3 K_{\theta}-B_{r} / 2 b^{2}-\frac{1}{2} \cos ^{2} \psi G_{r}\right]\right\}, \\
U_{y} \cos \theta^{\prime}=\cos \theta^{\prime}\left\{4 K_{\theta}(L-2)+4 K_{r}+2 \cos \psi \epsilon\left[K_{r}(1-L)+K_{\theta}(3-L)+B_{r} / b^{2}\right]\right\}, \\
0=2 \sin \theta^{\prime} \sin \psi\left\{\cos \psi G_{r}+\epsilon\left[\frac{1}{2} K_{r}(3-L)+K_{\theta}(L-2)+B_{r} / 2 b^{2}-\frac{1}{2} \cos ^{2} \psi G_{r}\right]\right\} .
\end{array}\right\}
$$

Clearly the stokeslet and doublet distributions can satisfy the boundary condition neglecting terms of $O\left(K_{l} \epsilon \ln \epsilon\right), l=r, \theta$, by taking

$$
\left.\begin{array}{l}
B_{r}=\frac{1}{2} b^{2} K_{r}, \quad K_{r}=(L-3) U_{y} / 2\left[\left(L-\frac{1}{2}\right)(L-2)-2\right], \\
K_{\theta}=\left(L-\frac{5}{2}\right) U_{y} / 4\left[\left(L-\frac{1}{2}\right)(L-2)-2\right] .
\end{array}\right\}
$$

The remaining velocity terms in (29) which do not satisfy the boundary condition may be written using the $\left(r_{1}, \psi, \theta\right)$ co-ordinate system as

$$
\mathbf{u}=\epsilon\left\{\sin \theta^{\prime}\left[D_{1} \mathbf{e}_{r_{1}}-D_{2}\left(\cos 2 \psi \mathbf{e}_{r_{1}}-\sin 2 \psi\left(\mathbf{e}_{\psi}\right)\right]-\cos \theta^{\prime} D_{3} \cos \psi \mathbf{e}_{\theta}\right\},\right.
$$

where $D_{1}=K_{\theta}, D_{2}=K_{\theta}+K_{r}, D_{3}=K_{r}\left(3 L-\frac{11}{2}\right)$. The first term above is a 'radial-like' flow in the cross-sectional plane and thus we introduce a potential source distribution (or equivalently a tangential doublet) into our solution. The second term is again an 'extensional-like' flow requiring a stresslet-quadrupole pair. The remaining term is a 'shear-like' flow with the shear gradient in the $\mathbf{e}_{r}$ direction and thus we introduce a rotlet whose strength is oriented in the $\mathbf{e}_{z}$ direction. The new velocity terms to be added to the original velocity field induced by the stokeslet and doublet distribution is therefore given by

$$
\left.\begin{array}{r}
\mathbf{u}=\int_{0}^{2 \pi}\left\{A \cos \theta \mathbf{U}_{\mathrm{r}}\left(\mathbf{R} ; \mathbf{e}_{z}\right)+B \sin \theta \mathbf{U}_{\mathrm{ss}}\left(\mathbf{R} ; \mathbf{e}_{r}, \mathbf{e}_{r}\right)+C \sin \theta \mathbf{U} \mathrm{q}\left(\mathbf{R} ; \mathbf{e}_{r}, \mathbf{e}_{r}\right)\right. \\
\left.+D \sin \theta \mathbf{U}_{\text {source }}(\mathbf{R})\right\} a d \phi \\
\mathbf{U}_{\text {source }}(\mathbf{R})=\mathbf{R} / R^{3},
\end{array}\right\}
$$

where

and the other singularities are given in (25b). The modulation of the singularity strengths by $\cos \theta$ or $\sin \theta$ is needed owing to the $\theta$ variation that appears in the velocity terms (31). Clearly we see that the total source strength is zero as it must be. 
Upon integration equation (32) gives the three velocity components on $r_{1}=b$ neglecting terms of $\left.O\left((A / b) \epsilon \ln \epsilon,(B / b) \epsilon \ln \epsilon,\left(C / b^{3}\right) \epsilon,(D / b) \epsilon \ln \epsilon\right)\right)$.

$$
\begin{aligned}
u & =2 / b \sin \theta^{\prime} \cos \psi\left[D+2 C / b^{2}+\cos 2 \psi\left(B-4 C / b^{2}\right)\right], \\
v & =2 / b \cos \theta^{\prime} A \cos \psi, \\
w & =2 / b \sin \theta^{\prime} \sin \psi\left[D-2 C / b^{2}+\cos 2 \psi\left(B-4 C / b^{2}\right)\right] .
\end{aligned}
$$

Consequently the boundary condition can be satisfied neglecting terms of $O\left(\epsilon^{2} \ln \epsilon\right)$

$$
\left.\begin{array}{l}
A=\frac{1}{2} b \epsilon K_{r}\left(3 L-\frac{11}{2}\right), \quad B=b \epsilon\left(K_{\theta}+K_{r}\right), \\
C=\frac{1}{4} b^{2} B=\frac{1}{4} b^{3} \epsilon\left(K_{\theta}+K_{r}\right), \quad D=-\frac{1}{2} b \epsilon K_{\theta} .
\end{array}\right\}
$$

The tangential doublet strength, which is equivalent to the source distribution, has a strength $\beta_{\theta}=-D a \cos \theta=\frac{1}{2} b^{2} \alpha_{\theta}$, and therefore we see that the frequently observed relationship between stokeslet and doublet is also true for this tangential doublet.

The principal result here is that the force per unit length in the radial and tangential direction is given by

$$
\left.\begin{array}{rl}
f_{r} & =\frac{4 \pi \mu[\ln (8 / \epsilon)-3] U_{y} \sin \theta}{\left[\ln (8 / \epsilon)-\frac{1}{2}\right][\ln (8 / \epsilon)-2]-2}+O\left(\epsilon^{2}\right), \\
f_{\theta} & =\frac{2 \pi \mu\left[\ln (8 / \epsilon)-\frac{5}{2}\right] U_{U} \cos \theta}{\left[\ln (8 / \epsilon)-\frac{1}{2}\right][\ln (8 / \epsilon)-2]-2}+O\left(\epsilon^{2}\right) .
\end{array}\right\}
$$

An interesting thing to note is the dependence of $f$ on $\epsilon$ which is distinctly different from the classical result for slender bodies where $f$ has always been taken to be proportional to $1 /(\ln \epsilon+$ constant). From the radial and tangential force per unit length (34) the total drag on the torus in the free-stream direction is found to be

where

$$
\begin{gathered}
F_{D}=2 \pi a \mu C_{D} U_{y}, \\
C_{D}=\frac{\pi\left(3 L-\frac{17}{2}\right)}{\left(L-\frac{1}{2}\right)(L-2)-2}+O\left(\epsilon^{2}\right) .
\end{gathered}
$$

Approximating this by neglecting terms of $O\left((1 / \ln \epsilon)^{3}\right)$ gives the result found by Cox (1970),

$$
C_{D}=\frac{3 \pi}{L+\frac{1}{3}}+O\left(\left(\frac{1}{\ln \epsilon}\right)^{3}\right)
$$

and thus we clearly see that the present result has summed the infinite series involving $(1 / \ln \epsilon)^{n}$.

\section{On edge rotation}

In this case the torus rotates with angular velocity $\omega_{x} \mathbf{e}_{x}$ (the results being equally applicable for $\omega_{y} \neq 0$ ). From (1), (2) and (3) we see that the no-slip condition at $\theta=\theta^{\prime}$ is

$$
\mathbf{u}=a \omega_{x}\left[\sin \theta^{\prime}(1+\epsilon \cos \psi) \mathbf{e}_{z}-\epsilon \sin \psi\left(\sin \theta^{\prime} \mathbf{e}_{r}^{\prime}+\cos \theta^{\prime} \mathbf{e}_{\theta}^{\prime}\right)\right] .
$$

Here we will omit the detailed discussion of constructing the solution since it proceeds exactly as before and simply note that in addition to the usual stokeslet and doublet 
distribution this problem requires a rotlet and stresslet-quadrupole distribution. The singularity strengths are taken to be (using notation consistent with the previous examples)

$$
\boldsymbol{\alpha}=K_{z} \sin \theta \mathbf{e}_{z}, \quad \boldsymbol{\beta}=B_{r} \sin \theta \mathbf{e}_{z}, \quad \boldsymbol{\delta}=A_{r} \cos \theta \mathbf{e}_{r}+A_{\theta} \sin \theta \mathbf{e}_{\theta},
$$

with stresslet and quadrupole strengths given by $B \sin \theta$ and $C \sin \theta$ together with the principal directions $\mathbf{e}_{r}$ and $\mathbf{e}_{z}$.

The velocity on the torus surface is found to be [neglecting terms of $O\left(\epsilon^{2} \ln \epsilon\right)$ ]

$$
\begin{aligned}
u= & 2 \sin \theta^{\prime}\left\{\cos \psi \sin \psi G_{z}+\sin \psi\left[K_{z} \frac{\epsilon}{2}(L-3)\right.\right. \\
& \left.\left.-\frac{\epsilon B_{z}}{2 b^{2}}-\frac{\epsilon}{2} \cos ^{2} \psi G_{z}+\frac{A_{\theta}}{b}+\frac{B}{b}+\frac{2 C}{b^{3}}+\cos 2 \psi\left(\frac{B}{b}+\frac{4 C}{b^{3}}\right)\right]\right\}, \\
v= & 2 \cos \theta^{\prime} \sin \psi\left[K_{z} \epsilon(2-L)+\frac{\epsilon B_{z}}{b^{2}}-\frac{A_{r}}{b}\right], \\
w= & 2 \sin \theta^{\prime}\left\{K_{z}(L-2)+\frac{B_{z}}{b^{2}}+\sin ^{2} \psi G_{z}\right. \\
& -\cos \psi\left[K_{z} \frac{\epsilon}{2}(L-3)+\frac{\epsilon B_{z}}{2 b^{2}}+\frac{\epsilon}{2} \sin ^{2} \psi G_{z}+\frac{A_{\theta}}{b}\right. \\
& \left.\left.-\frac{B}{b}-\frac{2 C}{b^{3}}+\cos 2 \psi\left(\frac{B}{b}+\frac{4 C}{b^{3}}\right)\right]\right\} .
\end{aligned}
$$

Thus the no-slip boundary condition becomes six equations for the six unknown strengths giving

$$
\begin{gathered}
K_{z}=\omega_{x} a / 2\left[\ln (8 / \epsilon)-\frac{3}{2}\right], \quad B_{z}=\frac{1}{2} b^{2} K_{z}, \\
A_{\theta}=\frac{3}{2} \epsilon(2-L) b K_{z}, \quad A_{r}=\epsilon b K_{z}, \quad B=\frac{1}{2} \epsilon b K_{z}, \quad C=-\frac{1}{8} \epsilon b^{3} K_{z} .
\end{gathered}
$$

The force per unit length is given by $-8 \pi \mu K_{z} \sin \theta$. In addition, the radial, tangential, and axial moment per unit length acting about the centre-line are found to be (see Johnson, 1977)

$$
M_{r}=-4 \pi \mu K_{z} b \epsilon \cos \theta, \quad M_{\theta}=4 \pi \mu K_{z} b \epsilon\left(3 L-\frac{11}{2}\right) \sin \theta, \quad M_{z}=0 .
$$

\section{Spinning torus}

Here we consider a torus rotating about its longitudinal axis with angular velocity $\omega_{z}$ and therefore the boundary conditions (1), (2) and (3) become at $\theta=\theta^{\prime}$

$$
\mathbf{u}=a \omega_{z}(1+\epsilon \cos \psi) \mathbf{e}_{\theta^{\prime}}
$$

For this case in which the body motion is completely tangential to the centre-line the solution does not require the use of a potential doublet distribution or a stressletquadrupole pair. The solution requires only a stokeslet and rotlet distribution which owing to the symmetry of the motion clearly have strengths given by

$$
\boldsymbol{\alpha}=K_{\theta} \mathbf{e}_{\theta}, \quad \boldsymbol{\delta}=A \mathbf{e}_{z} .
$$

Integration of the appropriately expanded integrands gives on the torus surface neglecting terms of $O\left(\epsilon^{2} \ln \epsilon\right)$

$$
u=w=0, \quad v=4 K_{\theta}(L-2)-\cos \psi\left[2 \epsilon(L-3) K_{\theta}-2 A / b\right] .
$$


Therefore the boundary condition is satisfied with

$$
K_{\theta}=\omega_{z} a / 4(L-2), \quad A=\epsilon b(3 L-7) K_{\theta} .
$$

Consequently the only non-zero components of the force per anit length and moment per unit length about the centre-line are

$$
f_{\theta}=-8 \pi \mu K_{\theta}, \quad M_{z}=-2 \pi \mu \epsilon b(6 L-13) K_{\theta} .
$$

\section{Expanding torus}

In this final case only $U_{r}$ in boundary conditions (1), (2), (3) is non-zero. The solution is constructed using a stokeslet $\left(\alpha=K_{r} \mathrm{e}_{r}\right)$, doublet $\left(\boldsymbol{\beta}=B_{r} \mathbf{e}_{r}\right)$, stresslet $\left(B \mathbf{U}_{\mathrm{ss}}\left(\mathbf{R} ; \mathbf{e}_{r}, \mathbf{e}_{r}\right)\right)$, quadrupole $\left(C \mathbf{U}_{\mathrm{q}}\left(\mathbf{R} ; \mathbf{e}_{z}, \mathbf{e}_{z}\right)\right)$ and a potential source $\left(D \mathbf{U}_{\text {source }}(\mathbf{R})\right)$. Since the torus is expanding (or contracting) clearly the source distribution is required to account for the change in body volume. Integrating to obtain the velocity field on $r_{1}=b$ gives (neglecting terms of $O\left(\epsilon^{2} \ln \epsilon\right)$ ),

$$
\begin{aligned}
& u=2 K_{r}(L-3)+\frac{2 B_{r}}{b^{2}}+2 \cos ^{2} \psi G_{r}+\cos \psi\left[\epsilon\left(\frac{7}{2}-L\right) K_{r}+\frac{2 D}{b}+\frac{4 C}{b^{3}}\right. \\
& \left.+2 \cos 2 \psi\left(\frac{B}{b}-\frac{4 C}{b^{3}}-\frac{1}{4} \epsilon G_{r}\right)\right] \text {, } \\
& v=0 \text {, } \\
& w=2 \sin \psi \cos \psi G_{r}+\sin \psi\left[\epsilon\left(\frac{1}{2}-L\right) K_{r}+2 \epsilon B_{r} / b^{2}+2 D / b-4 C / b^{3}\right. \\
& \left.+2 \cos 2 \psi\left(B / b-4 C / b^{3}-\frac{1}{4} \epsilon G_{r}\right)\right] \text {. }
\end{aligned}
$$

Thus the boundary condition is satisfied up to a term of $O\left(\epsilon^{2} \ln \epsilon\right)$ by taking

$$
\left.\begin{array}{c}
K_{r}=U_{r} / 2\left(L-\frac{5}{2}\right), \quad B_{r}=\frac{1}{2} b^{2} K_{r}, \\
D=\frac{1}{4} \epsilon b U_{r}, \quad B=-\varepsilon b K_{r}, \quad C=\frac{1}{4} b^{2} B .
\end{array}\right\}
$$

As expected $4 \pi$ times the total source strength equals the rate of change of the torus volume $\left(2 \pi^{2} b^{2} U_{r}\right)$. Additionally, the moments about the centre-line are found to be identically zero as anticipated from considerations of symmetry.

We note that the alternate problem produced by a line source located along the longitudinal axis of the torus is also solved by the above distributions with the following minor changes: (1) the source strength is zero $(D=0)$ since there is no change in torus volume; (2) $U_{r}=Q / a(1+\epsilon \cos \psi) \simeq Q / a\left(1-\epsilon \cos \psi+O\left(\epsilon^{2}\right)\right)$, where $Q$ is the line source strength; $(3)$ the stokeslet and stresslet strengths become

$$
K_{r}=(Q / a) / 2\left(L-\frac{5}{2}\right), \quad B=-\epsilon b\left(Q / 2 a+K_{r}\right),
$$

whereas the doublet and quadrupole strengths are given by equation (37) replacing $K_{r}$ and $B$ with those given in (38).

\section{Discussion}

We have presented the solution for the Stokes flow past a slender torus. The high degree of accuracy along with the convenient stepwise process in which the solution is constructed demonstrates how effective the method of flow singularities can be. Some general observations concerning the solution, which has served as a guide for the 


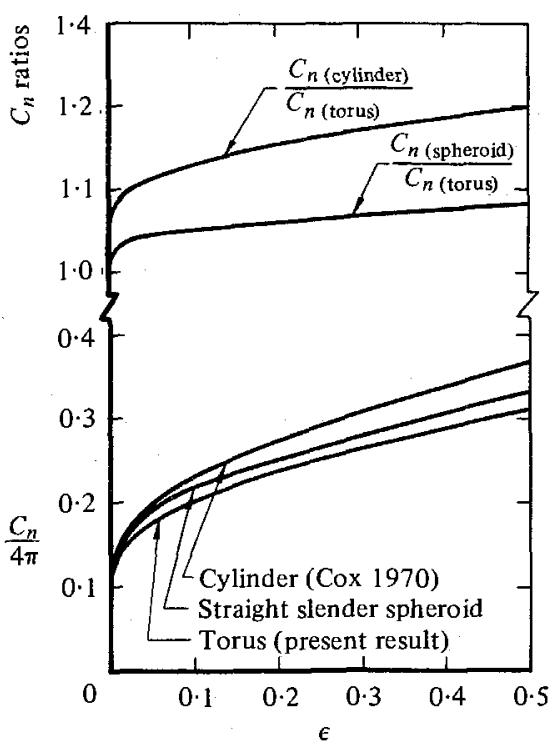

(a)
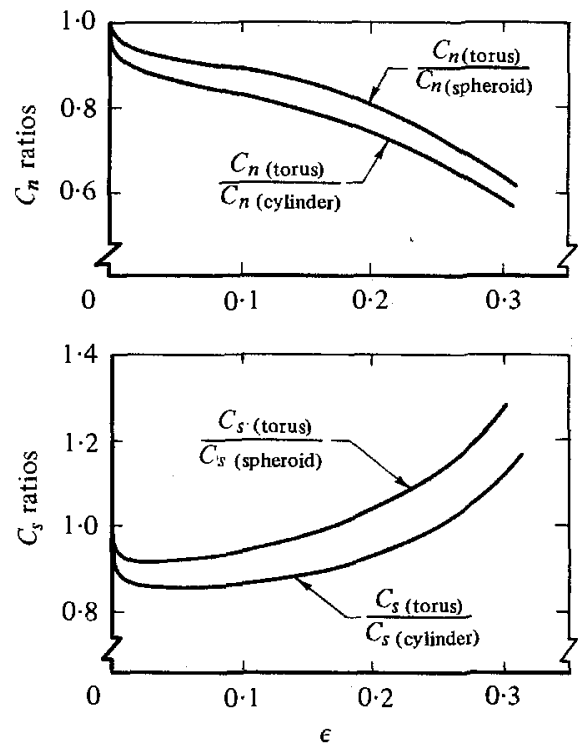

(b)

FIGURE 2. Drag coefficient comparison for: (a) broadwise translation;

(b) translation in the plane of the torus.

solution of slender bodies possessing arbitrary centre-line configurations, are worth noting at this time. Firstly, when satisfying the no-slip boundary condition up to an error term of $O\left(\epsilon^{2} \ln \epsilon\right)$ only a stokeslet distribution is required in the far field. The role of the higher-order singularities is to correct for the effects of local centre-line curvature and body shape. Furthermore, continuing the solution up to terms of $O\left(\epsilon^{2} \ln \epsilon\right)$ did not require modification of the stokeslet strength. In general, we found that a potential dipole and stresslet-quadrupole pair is needed whenever the body centre-line moves normal to itself. When the motion of the body centre-line is in its osculating plane (i.e. plane containing $\mathbf{e}_{r}, \mathbf{e}_{\theta}$ ) the solution invariably requires use of a rotlet distribution as does the case when the body is moving perpendicular to the plane in which the radius of curvature lies. If the solution is continued to one more term, namely satisfying the boundary condition through terms of $O\left(\epsilon^{2} \ln \varepsilon\right)$, a correction to the stokeslet strength of $O\left(\epsilon^{2}\right)$ is generally required. Such a small modification to the stokeslet strength or force is of little practical interest and therefore the details have been omitted.

More importantly, it is interesting to compare the force coefficients for the torus to those results found for straight centre-line slender bodies. This is particularly interesting since the straight body results have often been applied to bodies possessing finite centre-line curvature. We define the force coefficients in the directions normal and tangential to the centre-line as follows:

$$
C_{n}=f_{n} / \mu V_{n}, \quad C_{s}=f_{\theta} / \mu V_{\theta},
$$

where $n$ is either $r$ or $z$ depending on the case under consideration and $V_{n}, V_{\theta}$ are the components of the centre-line velocity. For example, in the case of translation in the plane we have $V_{n}=U_{y} \sin \theta, V_{\theta}=U_{y} \cos \theta$ and

$$
C_{n}=\frac{4 \pi(L-3)}{\left(L-\frac{1}{2}\right)(L-2)-2}, \quad C_{s}=\frac{2 \pi\left(L-\frac{5}{2}\right)}{\left(L-\frac{1}{2}\right)(L-2)-2} .
$$




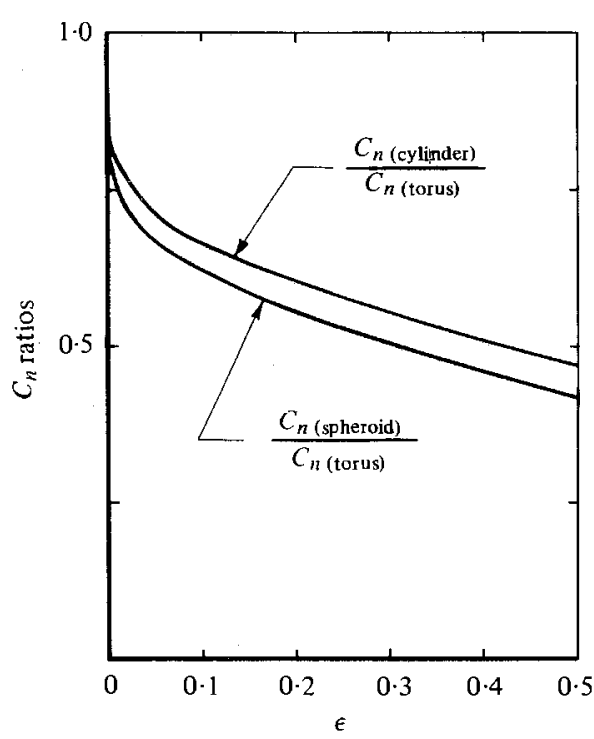

(a)

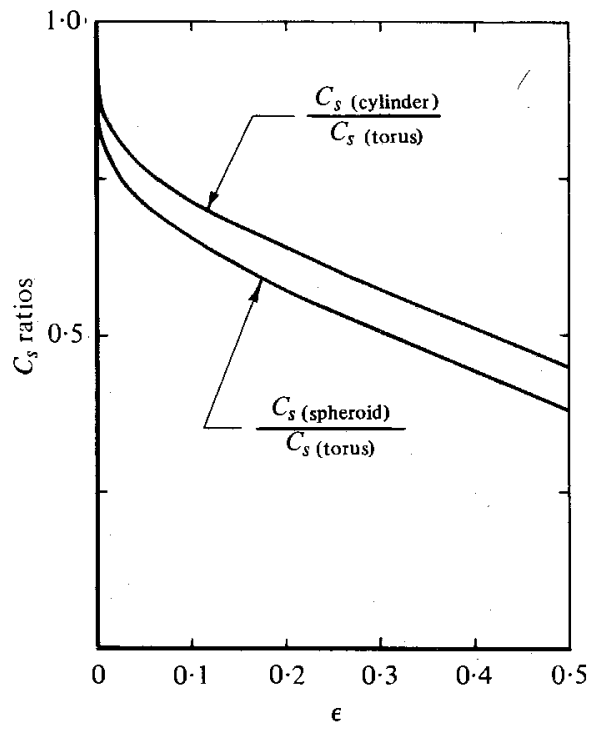

(b)

FiguRE 3. Drag coefficient comparison for: (a) on-edge rotation; (b) spinning.

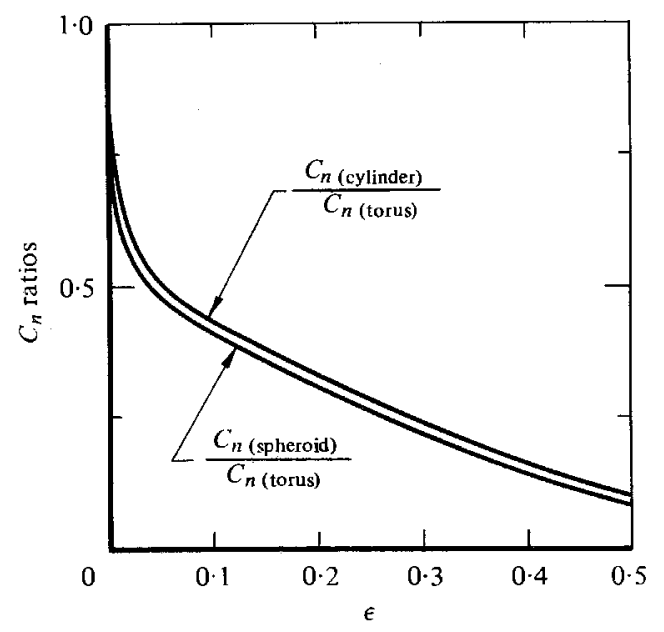

Figure 4. Drag coefficient comparison for an expanding torus.

In figures 2-4 we compare the force coefficients for the five torus motions to the force coefficients of (1) a straight slender spheroid

$$
C_{n}=\frac{4 \pi}{\ln (l / b)+\frac{1}{2}}, \quad C_{s}=\frac{2 \pi}{\ln (l / b)-\frac{1}{2}},
$$

and (2) a slender cylindrical body of constant cross-section (Cox 1970)

$$
C_{n}=\frac{4 \pi}{\ln (l / b)+0 \cdot 193}, \quad C_{s}=\frac{2 \pi}{\ln (l / b)-0 \cdot 807},
$$

where we consider bodies of equivalent length, i.e. $l=2 \pi a$. In figure 2 we have the two cases of torus translation, in figure 3 the two cases of rotation, and in figure 4 the 
case of an expanding torus. The comparison shows that while the difference is not large for the translational modes (less than about $15 \%$ for $\epsilon<0 \cdot 1$ ), it is quite large in the two cases of rotation and for an expanding torus. This may be attributed to the feature that in the latter cases the body centre-line velocity has finite variations, in magnitude and/or direction, along the torus. Considering the nature of Stokes flows, in which long-range effects are essential, such situations can clearly be expected to give considerable departures from the results found for straight bodies. In applications to micro-organism propulsion we may therefore expect that changes in phase along a flagellum or cilium in undulatory motion would have a similar effect on the force coefficients.

The simple geometry and availability of the present accurate analytic solution has also made the torus a particularly useful case for comparison between theory and experiment. Preliminary experiments are presently being developed.

\section{REFERENCES}

Chwane, A. T. \& WU, T. Y. 1974 Hydromechanics of low-Reynolds-number flow. Part 1. Rotation of axisymmetric prolate bodies. J. Fluid Mech. 63, 607-622.

Chwang, A. T. \& Wv, T. Y. 1975 Hydromechanics of low-Reynolds-number flow. Part 2. The singularity method for Stokes flows. J. Fluid Mech. 67, 787-815.

Cole, J. D. 1968 Perturbation Methods in Applied Mathematics. Waltham, Mass.: Blaisdell.

Cox, R. G. 1970 The motion of long slender bodies in a viscous fluid. Part 1. General theory. J. Fluid Mech. 44, 791-810.

Jonnson, R. E. 1977 Slender-body theory for Stokes flow and flagellar hydrodynamics. Ph.D. Thesis, California Institute of Technology, Pasadena, CA.

KapLUn, S. 1967 Fluid Mechanics and Singular Perturbations. Academic.

KeLLER, J. B. \& Rubinow, S. I. 1976 Slender-body theory for slow viscous flow. J. Fluid Mech. 75, 705-714.

VAN DYKE, M. 1975 Perturbation Methods in Fluid Mechanics. Standford, California: Parabolic. Wu, T. Y. 1976 Hydrodynamics of swimming at low Reynolds numbers. Contribution to Symp. Bewegungsphysiologie-Biomechanit, Akademie der Wissenschaften, Mainz, West Germany.

Wo, T. Y. 1977 Flagella and cilia hydrodynamics. Biomech. Symp. Yale University, New Haven, Conn.

Wu, T. Y. \& YATES, G. 1976 Finite-amplitude unsteady slender-body flow theory. 11th Symp. on Naval Hydrodyn., University College London. 